\title{
Generative feature-based design- by-constraints as a means of integration within the manufacturing industry
}

\author{
by Mark W. S. Jaques, John Billingsley and Dave Harrison \\ Portsmouth Polytechnic
}

This article examines the development of computer aids within manufacturing industry and proposes an altemative approach to the way we design and the designer's role within manufacturing. A featurebased generative design-by-constraints approach is applied, which requires the designer to specify solutions in terms of manufacturing data, which is captured by means of an interactive simulation of machining processes, in which the constraints of equipment, materials and tools are displayed to the designer. The effect of this approach on the integration of all areas within a manufacturing environment is explored, as is the simultaneous design nature of this approach.

\section{Introduction}

The history of computer-aided engineering is known to most readers although it is not often reviewed, mostly because people are preoccupied with the development and investigation of new ideas and solutions to the problem of integration of computer aids within the manufacturing industry. We feel that it is important when trying to develop methods of integration, that the reason for the lack of it should be explored.

Conventional CAD has developed from draughting packages, which were intended to speed up and improve the drawing process. The potential of 'digitally stored drawings' was soon appreciated. The first application was in component classification, an essential enabling part of group technology, where it was used to store large numbers of drawings in coded form, which would allow cross-referencing and hence the identification of similar components.

Similarly, CAM has developed from the use of computer controllers on machines, such as the 'plug board automatic lathe', to replace the board of sequencing switches and thus greatly reduce set-up times. The next application of digital drawings was in the generation of cutting tool paths for use on the newly developed computer-controlled machine tools. This was achieved by the addition of a cutting tool offset (tool radii) to the boundary of a drawing. These machines developed into the CNC machines we know today.

As the cost of computing power fell still further, another form of computer aid was developed to perform the costing and scheduling tasks known as 'computeraided planning and control'. Accountancy programs were developed to perform wages, costing and ordering functions. This ordering function developed into a separate program known as materials requirement planning (MRP). The program's function was to order automatically the components needed to construct an assembly. These programs were further developed to perform scheduling functions and became known as manufacturing resources planning packages (MRPII).

It can be seen from the descriptions of the origin of these computer aids that each was developed to automate and speed up an existing manual function, and that each was developed independently and without consideration for the others. This is an example of a nonsystems approach to the problem of computer-integrated manufacture, with the development of low-level sub-systems without first considering the system as a whole, leading to a corresponding incompatibility of input and output requirements.

\section{Approaches to integration}

Considerable research effort has been expended in an attempt to integrate the design and manufacturing functions [1-4]. as this is considered an essential part of flexible manufacturing systems. One approach is to use feature recognition to interrogate a CAD solid model of the design solution in order to identify manufacturing features, which are sets of information related to a part's description, such as holes and keyways. By analysing the geometric information within the solid model, and combining this with the feature information, manufacturing data are produced.

Three fundamental approaches have been identified by Shah and Rogers [2] for associating features with solid models. These methods are summarised below.

Human-assisted feature recognition

This approach has been used in preparing input for process planning systems, in which a planner could interactively mark surface types on a two-/three-dimensional 


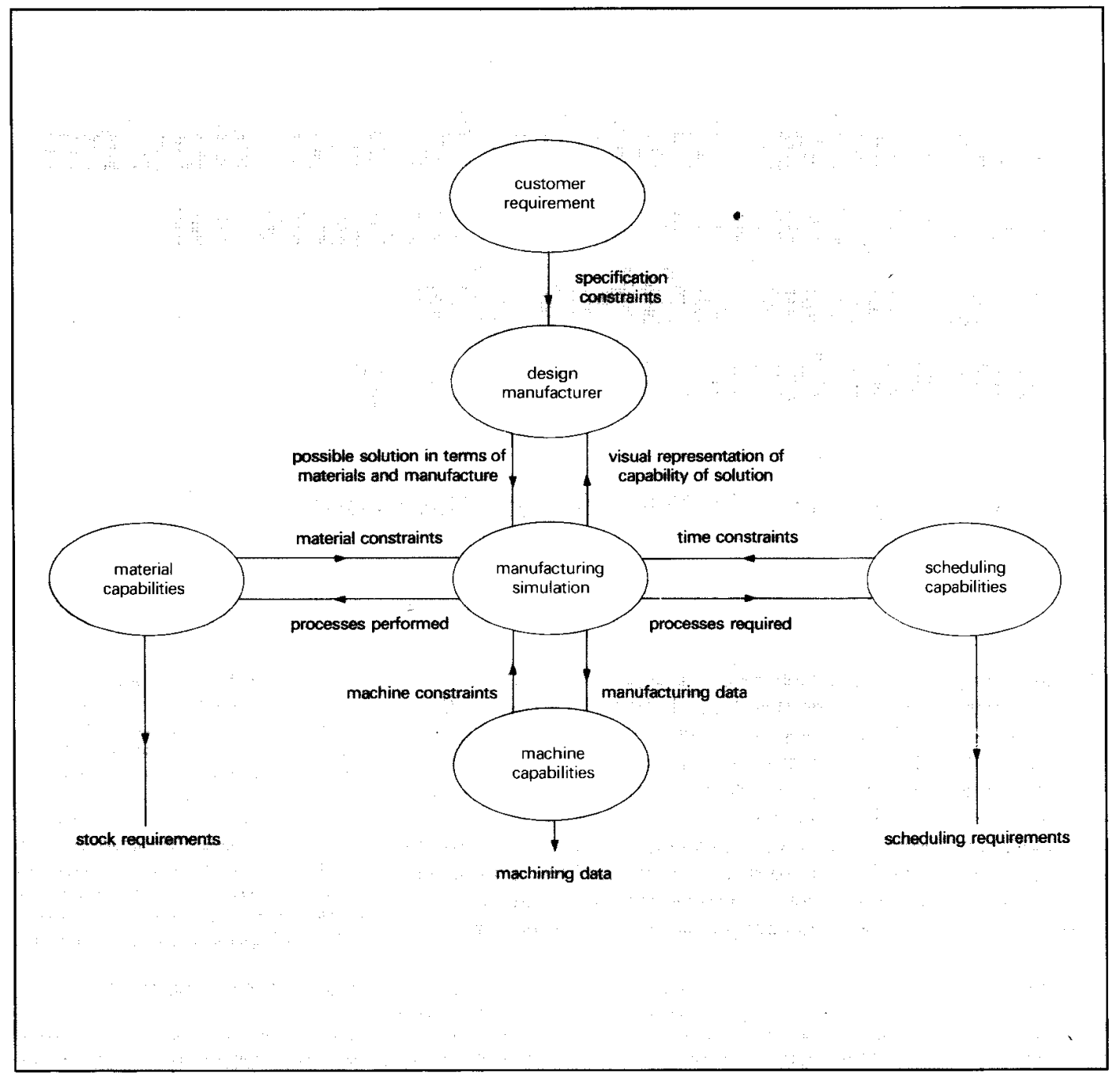

Fig. 1 DBC information and data-flow structure

image of the part; this allows geometry data from the modeller to be grouped as form features and to be combined with tolerance information. Other developers of process planning systems have also used this approach. It is clear that this method is neither convenient nor efficient, but was necessary in the absence of a better product definition method.

\section{Feature recognition and extraction}

In this approach, a feature recognition program examines the database produced by a solid modeller and makes deduc tions about the types of features present. The method amounts to making explicit what is implicit in the solid model. However, it cannot derive information that does not exist, such as tolerances and finish. There is also the possibility of misinterpretation. The algorithms for recognising even simple features are fairly complex and are generally modellerspecific. Feature recognition and extraction is redundant effort, which could be developed for retaining in the modeller all the information available to the designer

\section{Feature-based modelling}

This approach provides a means for building a complete database at multiple abstraction levels, right from the start of product development. Feature-based modelling systems allow users to build models using features stored in libraries and to network these together as needed.

Previous feature-based approaches give rise to a number of fundamental problems: information cannot be extracted i it is not present, for example, surface finish and tolerances; some surfaces or volumes cannot be uniquely segmented into features [5]; construction of a model by using features limits designers to those features which exist in the library, and thus restricts their creativity. Information about how a part is to be manufactured is not present in a solid model; for instance, a hole may be drilled in one operation, it may be milled using circular interpolation, it may be stamped or cut out, and the particular process used will affect the performance of the components. Yet, there is no way of telling from the solid model which method is to be used.

Process planning systems have been proposed as a method of integrating design and manufacture. There are again three main approaches identified by Pande and Palsule [3]. 


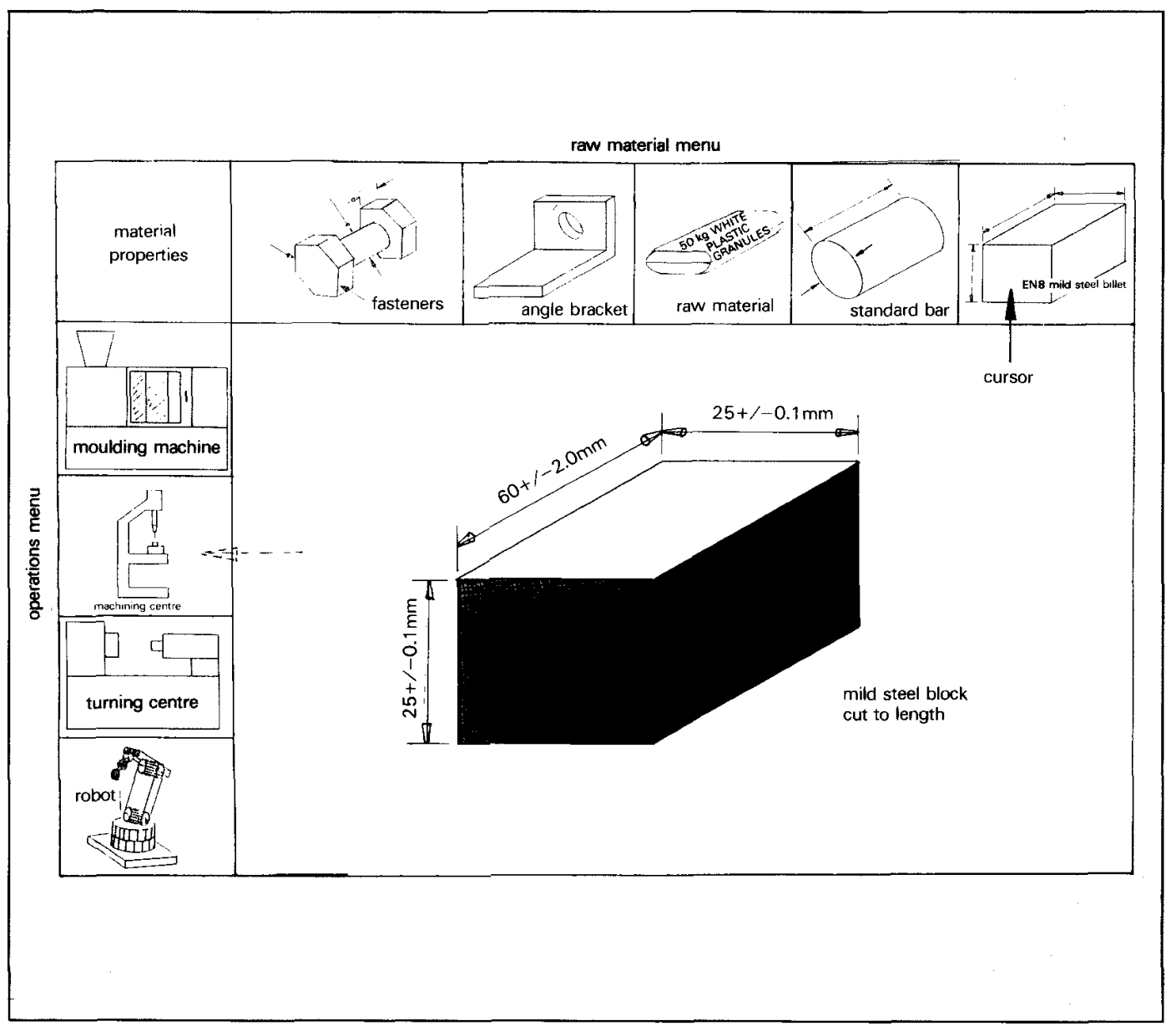

Fig. 2 Selection of raw material

The variant approach

These systems basically provide storage and retrieval of standard process plans for part families, which have been created by the classification of parts using a group technology approach. These predefined parts are assembled to form the required component, along with the required process information. The major disadvantage of the variant approach is its inflexibility and the unrealistic constraints that it places on the designer.

The generative approach

Generative systems are more fundamental in nature, as they provide interactive part generation, technology strategies and decision logic for operation planning. A question-and-answer session is used to lead a designer through a menu of allowable operations, extracting manufacturing data as it progresses. Thus, a component is described in terms of its method of manufacture. The main disadvantage of this approach is that it is not a form of representation normally used by designers.

The knowledge-based expert system

This type of system is similar to the feature recognition approach described previously and has similar problems. It forces the designer to work within the expert's restrictions. It can be used in an advisory role, informing the designer of conventional methods and restrictions.

An unusual method of integration is to make the manufacturing system so flexible that it can accept solid model information direct from a CAD system, and thus produce the product.

Such a manufacturing method is that of stereolithography [6], where a laser scans the surface of a bath of liquid (acrylic resin), slowly constructing the part layer by layer. This process has potential, especially in the product realisation and testing stages, but the materials that can be used are very restricted at present and the equipment is not yet used in industry.
Previous approaches have had limited success; either requiring input from a process planner after the design solution has been provided, or being applicable to only a restricted family of components or processes. None of the previous methods of integration will work in isolation, as they all approach the problem from a particular area and attempt to join separately developed sub-systems. Thus, they are not a cure to the problem of integration but are merely approaches to solving the symptoms of a lack of integration.

\section{A new approach}

A new approach to the way we design and the designer's role is required, not based on the development of any one particular sub-system, but based on an appreciation of the system as a whole. It is easiest to define this approach by looking at a simple example of a manufacturing system.

The gardener analogy 


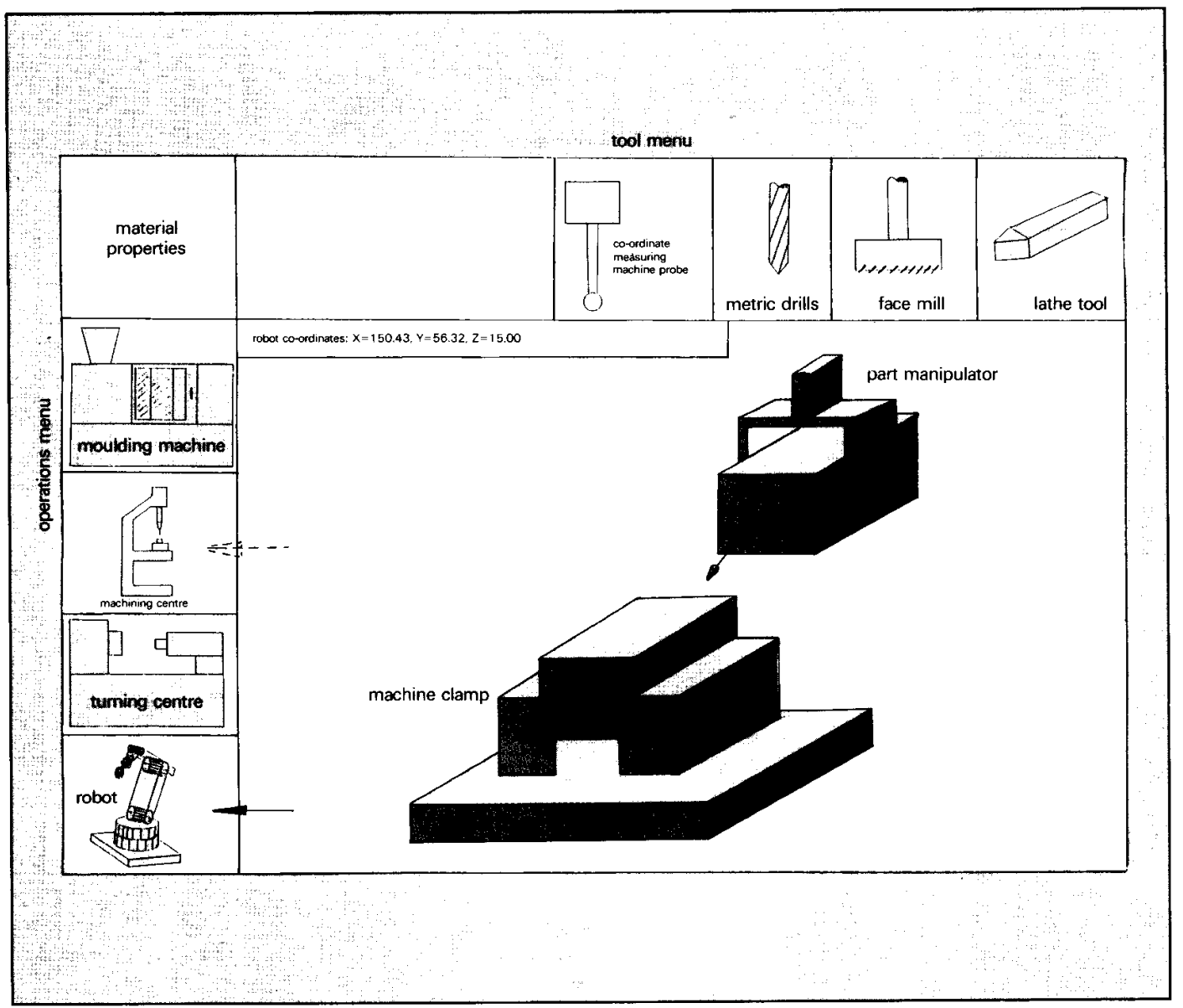

Fig. 3 Manipulation and clamping of work piece

A gardener wants to mow the lawn, but cess are not compatible, they will go has lost the grass collection box. The gar- through the assessment again until they dener decides to make a new grass box so have a suitable combination or realise that the grass cuttings will not have to be that the task is beyond their capabilities. raked up. What is the sequence of events that the gardener follows to arrive at the required output, a grass box?

Does the gardener start by modelling several possible solutions in threedimensions, then produce engineering drawings of the solution, specifying such things as colour, surface finish and tolerances? Does the gardener then sit down with a book of synthetics and work out the time it will take to manufacture and the most efficient sequence in which to perform the operations? The answer is no, because if the gardener follows this route, it will become apparent that neither are the materials available nor the equipment to produce the design.

What the gardener really does is to go to the garage to see what materials are available. The gardener looks at the available equipment and, with this knowledge, determines which processes and which materials to use. If the material and pro- ment, materials and tools are displayed to the designer.

\section{The methodology}

The designer/manufacturer selects, from the available raw materials, information about mechanical properties, and tolerances are displayed on the screen, along with a representation of the raw material. A machine tool with a well defined repertoire of operations is then selected. The user might define a path for a pass by an end-mill. The effect of such a cut would be shown on the display, together with an appropriate warning if machine or material constraints were being exceeded; for instance, the cutter may be in danger of damage. The effect of the cut on changes in tolerance, surface finish and mechanical properties, as a result of the machining process, are also displayed. As 'surrogate' machining operations are progressively entered, the shape of the remaining component would be defined, producing a three-dimensional representation of the component as a by-product. This mode 


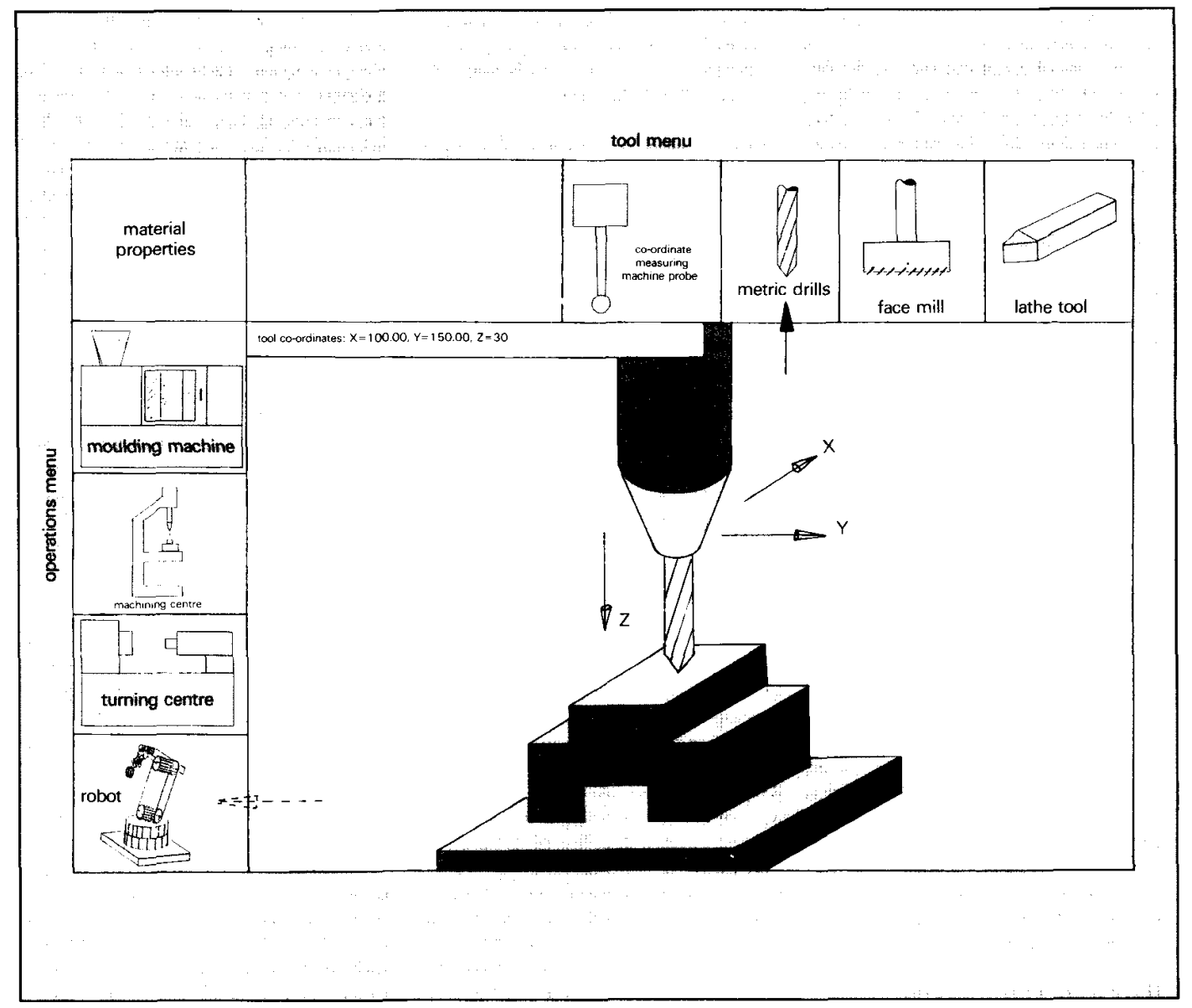

Fig. 4 Performing the drilling operation

is then available for analysis by conventional computer-aided testing packages. When the design has been completed to the satisfaction of the user, the operations can be 'replayed' on the machine tool itself, resulting in the cutting of the actual component.

This approach can be applied not just to machine tools, but also to materialshandling devices, such as robots and conveyors, which can be simulated and manipulated on the screen. Inspection equipment, such as co-ordinate measuring machines, can also be simulated and the method of inspection defined.

Manufacture as a means of design

Manufacture has not been used as a means of design since the days of the village blacksmith. Blacksmiths had an expert knowledge of the capabilities and limitations of their equipment and materials. A customer would come to the blacksmith with a requirement. The black smith would then make a solution to that problem; if the first attempt did not work,

Computer-Aided Engineering Journal

it would then be modified or another one manufactured. The design problems were simple enough for one person to solve, but as customer requirements became more complex, so the marketing, design and manufacturing functions were carried out by separate people, and thus designby-manufacture ceased.

Graphic designers' (who can be regarded as two-dimensional manufacturers) tasks rarely became more than a single person could cope with, and so the method of design remained one of manufacture. The designer cuts, pastes and paints until a satisfactory solution has been manufactured. With the advent of the computer, this process has become automated. This resulted in speeding up the task and greatly reduced the cost, as well as reducing its complexity; allowing inexperienced people to perform their own graphic design and also allowing graphic designers to tackle more complex tasks. The addition of computing power was to the whole system, not to any individual sub-system, as in the case of manufacturing, accounting for the suc- cess of computer-aided graphic design.

This approach is to be applied to 3-D manufacturing, in the hope that it will integrate all areas, and allow a single person to control a FMS cell and produce a product as easily as a graphic designer can with a printer or plotter.

\section{Design-by-constraints}

Specifying constraints, rather than capabilities, leads to concise specifications of systems which gives greater freedom. An analogy can be made with the law, which specifies only what is illegal, thus allowing people to do anything which is not unlawful. Whereas to specify all that is lawful would require considerable information storage and would restrict the individual. This reduced information storage and data-flow requirement of the design-by-constraints (DBC) approach leads to the inforrilation structure shown in Fig. 1. With generative feature-based design-by-constraints, the means of input is by actual manipulation of tools on the screen and machining simulation (the 
generation of manufacturing features) The only limitations are those of the machine, its physical motion restriction its tool library, dimensional, scheduling and tolerance capabilities. These are the true restrictions that the designer works within. Thus, the component designed can be manufactured.

\section{Hole specification}

An example of how the proposed design method would function is best demonstrated by describing how the system would represent a simple hole.

The user selects a raw material, from the list of available materials, whose dimensions are known. The user then selects an appropriate machine and tool from those available (Fig. 2). The raw material is then moved from the materials handling device to the machine surface. The means and method of position and clamping is carried out on the screen by the user (Fig. 3). The machine tool's speed and feeds are specified by the user, or can be selected by the program with its knowledge of machine, material and too type that the selection and loading process has implied. The tool is moved interactively on-screen to the point where the hole is to be drilled. The drill is then brought down by the user in the number of pecks desired. If the speed is too high, the simulation can warn the use (Fig. 4). Once the hole is drilled, the component is unloaded and placed on the materials-handling system.

Other work in the area of manufacturing simulation

In 1975, Gossard [7] developed an analogic approach to NC part programming. He used hand cranks, similar to those used to control the slides of manua lathes, to control the position of a too displayed via interactive graphics on a computer screen. The work centre around two-dimensional tuming operations The dynamic display of the working area included not just tool and work pieces, but also fixturing and clamping devices As the tool is moved by the programmer so the corresponding part shape change is displayed. The tool co-ordinates are displayed along with cut conditions, auxiliary functions and tool information. This information is used to generate NC tape. This approach was entitled 'part programming by doing'. Gossard suggested that this analogic approach could also be used as a means of design. Gossard described the term analogic as

'motion conveyed in analog form by moving a joystick or turning a handle with a computer graphic display providing visual feedback of the work piece, fixturing, the cutting tool and its position. The operator can produce the part as if he were machining it in a conventional fashion'.

Gossard's work was expanded to three dimensional machining by the CADCentre in its package GNCplus [8], which uses the design-by-control-of-tool-motion approach as an addition to its tool-path simulation capability and which was intended to be used in an interactive edit mode.

Encee systems produced a package called SmartCam [9], which also uses the design-by-tool-motion approach and is capable of performing milling, turning, wire EDM and punch press applications. Another package from Encee systems, called Vericut, performs similar functions to that of GNCplus.

Although the previous packages use a design-by-simulation approach, they consider the machine tool in isolation. Thus, no information regarding materials handling, part manipulation, tooling, material availability or scheduling is generated by these simulations.

There are other packages that use simulation to generate the missing information mentioned above. CRASP from BYG uses the simulation of robot motions as a means of planning robot tasks; for instance, the unloading of a machined part from a machining centre. More recently, a simulation-based scheduling system has been developed for Israel Aircraft Industries (IAI) [10], which uses a simulation of possible paths through a factory in order to determine the most efficient. These systems each consider only an element of the manufacturing process and are not simulating manufacture as a whole, and therefore do not aid the integration of manufacturing. Each of the previous simulations packages carry out the same basic task, and it is for this reason that a single package capable of simulating the entire manufacturing process can be contemplated.

\section{The advantages of the DBC approach}

\section{Group working}

As the tasks or projects become too large for a single person, then multidisciplinary groups are formed, as suggested by Putnam [11]. These groups are formed at the start of a design project, as the design stage is the area where the majority of costs are determined. The DBC approach is ideal for a multidisciplinary approach, as the system is not design-based but manufacturing-based, thus allowing all areas to see the implications of their specification on the cost, lead time and quality of the product.
The production planner can see the extra loading that will occur from the design, and thus can make the part using a different machine which is less loaded, thus reducing lead times. The costing implications are apparent in terms of manufacturing time, which also gives delivery dates. Thus, the effects of changing the standard of the raw material, e.g to a cheaper one, can be seen in terms of the cost changes in machining and quality implications. Hence, the true saving can be determined. The designer's role becomes one of managing the group.

\section{Simultaneous design}

The limitations and constraints of the equipment and materials, along with quality, cost etc., can all be considered simultaneously by the group and the interdependent nature of their skills becomes apparent. Thus, the old-fashioned approach of design first, ignoring the constraints, leaving the other departments to sort out the problems (usually at high cost), has been replaced by a simultaneous multidisciplinary approach, as proposed by Black [4].

This approach reduces the total product lead time and reduces the product cost, both in terms of time and also in terms of the cost-determining nature of the early stages of design. As stated by Whitney [12], 80\% of product cost is determined in the design stage. Yet, in traditional manufacturing, $80 \%$ of resources and people are expended in trying to reduce the last $20 \%$ of costs which can be determined in the manufacture stage. The DBC approach expends $100 \%$ effort in the design stage of a product, with the manufacture being automatic.

\section{Tolerances}

The design-by-constraints approach also has implications for the way designers use and specify tolerances. Consider a simple machining operation. The initial raw material has had its mechanical properties and dimensional distributions specified during the commissioning stage, and thus has a tolerance band which is known to the computer and can be displayed graphically to the designer. Once selected, the raw material is clamped to a machine bead. Again the tolerance limitations of the equipment are known to the program, and hence the cumulative tolerance of both clamping device and raw material is known.

Next, a tool is selected which also has a tolerance, as does the tool holder and machine slide (motion). All these tolerances are predefined, and the total tolerance of the final part is thus known. The part has been designed to true tolerances. A distribution of part sizes or a fuzzy boun- 
dary to the part can be displayed. If all components are designed in the same way or the tolerance is known, a probability of assembly can be generated.

Thus, the quality implications of the design have been highlighted. Tolerances are no longer specified without their cost quality and lead time implications being considered. Truslove [13] said that

The use of CAD and its integration with CAM will lead to the design function becoming more accountable for any errors that arise in the product definition'.

There has to be total product simulation including tolerance analysis, of com ponents before any manufacture is attempted. Tolerances should be applied and analysed at the initial stages of design, hence giving a better indication of the effect that tolerances would have on the overall design. Truslove states that

Any benefits in manufacture caused by increasing the amount of analysis carried out during the design stage should be attributed to the overall design manufacturing process, rathe than individual activities

It is believed that design by the simulation of manufacturing constraints fulfills this task.

\section{Distribution manufacturing environments}

Another advantageous area of application of the DBC approach is within companies which have a distributed manufacturing environment, with several factories in different countries, each with different manufacturing constraints. With the design-by-manufacture method, a part designed centrally can have its method of manufacture changed to best suit each factory and better utilise their resources

\section{The size of the task}

The implementation of the ideas demonstrated here present a monumental task both in computing and manpower required. For the above reason, research will concentrate on one small manufacturing cell in order to enable the demonstration of the proposed methodology. Even this limited demonstration of the design-by-constraints approach will require the utilisation of transputer technology to create the necessary computing powe and parallel processing capability.

\section{Proposed application of DBC}

The collaborating company on this project is TRW United Carr, who produce a large range of small plastic components for the automotive industry both in Europe and the USA. In order to test the theory of DBC, a flexible manufacturing system consisting of a conveyor, several robots, a machining centre and a small moulding machine, is being assembled in the Robotics Department at Portsmouth Polytechnic. This will be used as a test bed for the DBC approach, and it is hoped that it will allow us to investigate the approach and answer questions such as the following.

- Can the approach be implemented in a modular form?

- What happens to the system if tool library, stock levels or machine loading are not those that have been reported? - What are the qualitative and quantitative advantages of this approach ove conventional jobbing shops, CAD/CAM FMS or batch production?

- How are constraints to be collected specified, stored and maintained?

- How large will the computational requirements of this approach be when applied to a small manufacturing company?

- What are the quality implications of the DBC approach?

- Can the approach work without direct numerical control of machine tools?

How can we model humans in terms of constraints?

- Are there other areas of application of the DBC approach, such as electrical or civil engineering?

\section{Conclusion}

The application of a systems approach to the area of manufacturing has given rise to a new approach to design and the designer's role. Generative feature-based design-by-constraints is a method that allows the simultaneous generation of all information required to satisfy a customer requirement by the production of an artifact. It thus integrates all areas of manufacturing, giving each area an appreciation of its role in the design process. The conceptual nature of design still exists. This approach has changed the method of representing and developing these concepts in an environment where all implications of the design can be assessed at the earliest stage.

\section{Acknowledgments}

The authors would like to thank Terry Oliver and TRW United Carr for their technical and financial support, which will allow us to continue and expand this project.

\section{References}

[1] CHOI B.K., and BARASH, M.M.: 'STOPP: an approach to CADCAM integration', Comput. Aided Des. 1985, 17, (4), pp. 162-168

[2] SHAH, J.]., and ROCERS, M.T.: 'Functional requirements and conceptual design of the feature-based modelling systems', Comput-Aided Eng. J., 1988, 5, (1), pp. 9-15

13] PANDE, S.S., and PALSULE, N.H.: 'GCAPPS-a computer-assisted generative process planning system for turned components', Comput.-Aided Eng. J., 1988, 5, (4) pp. 163-168

[4] BLACK, 1.: 'Embodiment design: facilitating a simultaneous approach to mechanical CAD'. Comput.-Aided Eng. J., 1990, 7, (2), pp. 49-53

[5] WOODWARK, J.: 'Shape models in computer integrated manufacture-a review', Comput.Aided Eng. J., 1988, 5, (3), pp. 103-112

[6] JAMIESON, R.: 'New options in aerodynamic modelling: CAD, CFD, stereolithography and shading in a workstation environment'. Comput-Aided Eng, f., 1990, 7, (3), pp. 75-79

[7] GOSSARD, D.C: 'Analogic part programming with interactive graphics'. MIT, PhD Thesis, 1975

[8] CADCENTRE.: 'Addition to GNC package'. Comput.-Aided Eng. J., 1990, 7. (6), pp. 185

[9] ENCEE SYSTEMS.: 'SmartCAM'. Point Control Co., Eugene, Oregon, USA, 1989

[10] FEUER, Z., GIVON, R., and DAR-EL, E.: 'A simulation-based scheduling system', FMS Mag.. 1989, 7. (1), pp. 15-17

[11] PUTNAM, A.O.: 'A redesign for engineering', Harv. Bus. Rev., 1985, pp. 139-144

[12] WHITNEY, D.E.: 'Manufacturing by design', Haw. Bus. Rev., 1988, (4), pp. 83-91

[13] TRUSLOVE, K.C.E.: 'The implications of tolerancing for computer-aided mechanical design', Comput.-Aided Eng. f., 1988, 5, (2), pp. 79-85

M.W.S. Jaques, a research scientist, Professor j. Billingsley, Head of Robotics, and D. Harrison are with the School of Systems Engineering, Portsmouth Polytechnic, Anglesea Road, Portsmouth PO1 3DJ. 